\section{Estudo retrospectivo do mastocitoma cutâneo canino utilizando duas classificações histopatológicas}

\author{
Retrospective study of canine mast cell tumor using two \\ histopathological classification
}

\begin{abstract}
Anna Paula Martins de Carvalho ${ }^{1 *}$, Gabriella Massine Nogueira², Hassan Jerdy Leandro ${ }^{3}$, Raphael Mansur Medina ${ }^{3}$, Eulógio Carlos Queiróz de Carvalho', Andrigo Barbosa de Nardi \& Leonardo Serafim da Silveira'

'Substituir por: Médicos veterinários, DSc. Laboratório de Morfologiae PatologiaAnimal-LMPA, Centro de Ciênciase Tecnologias Agropecuárias - CCTA, Universidade Estadual do Norte Fluminense Darcy Ribeiro - UENF, Campos dos Goytacazes, RJ, Brasil ${ }^{2}$ Médica veterinária. Laboratório de Morfologia e Patologia Animal - LMPA, Centro de Ciências e Tecnologias Agropecuárias CCTA, Universidade Estadual do Norte Fluminense Darcy Ribeiro - UENF, Campos dos Goytacazes, RJ, Brasil

${ }^{3}$ Médicos veterinários, MSc. Laboratório de Morfologia e Patologia Animal - LMPA, Centro de Ciências e Tecnologias Agropecuárias - CCTA, Universidade Estadual do Norte Fluminense Darcy Ribeiro - UENF, Campos dos Goytacazes, RJ, Brasil ${ }^{4}$ Médico veterinário, DSc. Departamento de Clínica e Cirurgia Veterinária, Faculdade de Ciências Agrárias e Veterinárias, Universidade Estadual Paulista Júlio de Mesquita Filho - UNESP, Campus Jaboticabal, Jaboticabal, SP, Brasil
\end{abstract}

\section{Resumo}

O número de neoplasias relatadas em cães vem aumentando a cada ano e o mastocitoma representa o tumor cutâneo mais comum. Existem poucos estudos descrevendo o perfil dos animais acometidos por processos neoplásicos no Brasil. Este trabalho teve como objetivo realizar um estudo retrospectivo dos animais com mastocitoma atendidos no Hospital Veterinário da UENF. Foram avaliadas características como sexo, raça, idade, localização tumoral e o grau histológico pelas classificações de Kiupel e Patnaik de 61 animais. Os machos representaram a maioria (59\%) dos animais e pacientes idosos com idade entre 7 e 12 anos, foram os mais acometidos. No que diz respeito ao padrão racial, os, cães sem raça definida, cães da raça Boxer, Labradores e Pinscher foram os mais observados. A região de membros (38,5\%), seguida por tumores em tronco (27\%) e tumores genitais (13,1\%) foram os locais mais acometidos. Os animais com tumores múltiplos também foram relatados (13,4\%). Seis tumores foram classificados como grau I, 45 como grau II e 10 como grau III. De acordo com a classificação de Kiupel, a maioria dos tumores (68,85\%) foi classificada como de baixo grau. Pelo presente estudo pode-se observar que os animais idosos foram os mais acometidos, a raça e a localização estão relacionadas ao grau histológico e os mais comuns foram os graus II e o baixo grau.

Palavras-chave: cão, histopatologia, tumor de mastócitos, Patnaik, Kiupel.

\begin{abstract}
The number of reported cancer in dogs is increasing every year and the mast cell tumor is the most common skin tumor. There are few reports regarding tumor characteristics in Brazil. This study aimed to conduct a retrospective study of animals with mast cell tumors treated at the Veterinary Hospital of UENF. Evaluation of gender, breed, age, tumor location and histological grade by Kiupel and Patnaik was ratings of 61 animals. Males were majority (59\%) of animals and elderly patients aged between 7 and 12 years were the most affected. Most dogs were mixed breed, Boxer, Labrador and Pinscher. The region members (38.5\%), followed by tumors in the trunk (27\%) and genital tumors (13.1\%) were the most common tumor localization. Animals with multiple tumors were also reported (13.4\%). Six tumors were classified as grade I, 45 grade II and 10 as grade III. According to Kiupel classification, most tumors (68.85\%) were classified as low grade. By this study it can be seen that the older animals were the most affected, race and location are related to the histological grade and the most common were the grade II and the low grade.
\end{abstract}

Keywords: dog, histopathology, mast cell tumor, Patnaik, Kiupel.

\section{Introdução}

A alta incidência de neoplasias na espécie canina está associada com a maior longevidade destes animais, o que os expõe a carcinógenos por mais tempo e a um sistema imune comprometido (Withrow \& Vail, 2007). \section{BJ $\mathbb{M}$ \\ Brazilian Journal of Veterinary Medicine \\ p-ISSN 0100-2430 \\ e-ISSN 2527-2179 \\ ○}

Como citar: Carvalho, A. P. M., Nogueira, G. M., Leandro, H. J., Medina, R. M., Carvalho, E. C. Q., Nardi, A. B., \& Silveira, L. S. (2017). Estudo retrospectivo do mastocitoma cutâneo canino utilizando duas classificações histopatológicas. Brazilian Journal of Veterinary Medicine, 39(3), 170-175. doi: 10.29374/2527-2179.bjvm027316

Fonte de financiamento: Coordenação de Aperfeiçoamento de Pessoal de Nível Superior (CAPES).

Conflito de interesses: Os autores declaram não haver conflito de interesses que precisam ser informados.

Recebido: Fevereiro 01, 2016

Aceito: Julho 22, 2016.

O estudo foi realizado na Universidade Estadual do Norte Fluminense Darcy Ribeiro - UENF, Campos dos Goytacazes, RJ, Brasil.

\section{*Correspondência}

Anna Paula Martins de Carvalho Laboratório de Morfologia e Patologia Animal - LMPA, Centro de Ciências e Tecnologias Agropecuárias - CCTA, Universidade Estadual do Norte Fluminense Darcy Ribeiro - UENF Av. Alberto Lamego, 2000

CEP 28035-200 - Campos dos Goytacazes (RJ), Brasil

E-mail: annapmcarvalho@gmail.com

Copyright Carvalho et al. Este é um artigo publicado em acesso aberto (Open Access) sob a licença Creative Commons Attribution Non-Commercial, que permite uso, distribuiç̧ão e reprodução em qualquer meio, sem restrições desde que sem fins comerciais e que o trabalho original seja corretamente citado. 
O mastocitoma é o tumor cutâneo mais comum em cães (London \& Seguin, 2003; Withrow \& Vail, 2007), representando 16 a 21\% dos tumores cutâneos na espécie (Daleck et al., 2008). Pode acometer a derme ou o tecido subcutâneo com diversas apresentações, o que pode dificultar estimativas do comportamento tumoral (London \& Seguin, 2003; Strefezzi et al., 2003).

Acomete, principalmente, cães entre oito e nove anos (London \& Seguin, 2003), mas pode acometer animais entre um ano e meio e dezessete anos (Costa-Casagrande et al., 2008) e não há predileção por sexo (Costa-Casagrande et al., 2008; Furlani et al., 2008). A literatura descreve uma maior predisposição para a ocorrências destas neoplasias em cães das raças Boxer (Costa-Casagrande et al., 2008; Furlani et al., 2008), Boston Terrier, Bulldog inglês (Goldschmidt \& Hendrick, 2002; London \& Seguin, 2003), Labrador, Golden Retrievers, Cocker Spaniel, Schnauzer, Teckel e Shar-pei (Daleck et al., 2008). Cães mestiços também possuem alta incidência de mastocitoma, o que pode ser explicado pelo grande número de cães sem raça definida atendidos na rotina da clínica veterinária de determinadas regiões (Costa-Casagrande et al., 2008; Furlani et al., 2008).

O mastocitoma cutâneo se localiza, frequentemente, nas regiões do tronco e regiões perineal e genital (50-60\% dos casos) (Furlani et al., 2008), seguida dos membros (25\%) (Govier, 2003), mas também podem aparecer com certa frequência na cabeça e no pescoço (Kiupel et al., 2005; Fulcher et al., 2006). Ainda não há estudos conclusivos de que o tumor em determinadas regiões, como junções muco-cutâneas, sejam mais agressivos do que em outras (London \& Seguin, 2003).

A graduação histopatológica é o método de eleição para prever o comportamento biológico do mastocitoma (Patnaik et al.,1984) e, atualmente, são usadas duas classificações: a de Kiupel et al., (2011) que os divide em dois graus de malignidade e a de Patnaik et al. (1984) que os divide em três graus de malignidade.

O objetivo deste trabalho foi realizar um estudo retrospectivo para analisar a frequência de animais acometidos por mastocitoma cutâneo, atendidos no Hospital Veterinário da UENF, bem como avaliar os dados epidemiológicos a respeito da raça, idade, sexo e localização.

\section{Material e métodos}

Foram diagnosticados 61 mastocitomas pela avaliação histopatológica e observadas a idade no diagnóstico, o sexo, a raça e a localização tumoral descritas no prontuário de atendimento. A apresentação tumoral foi considerada disseminada naqueles pacientes que apresentavam mais de um tumor e os animais foram divididos em cinco faixas etárias (0-3 anos, 4-6 anos, 7-9 anos, 10-12 anos e maiores que 12 anos). Os tumores localizados na bolsa escrotal, no períneo e na vulva foram agrupados e classificados como tumores genitais para efeito de avaliação.

O diagnóstico foi realizado pela análise histopatológica baseado nas classificações de Kiupel et al. (2011) e Patnaik et al. (1984).

Os critérios utilizados para a graduação tumoral, alto ou baixo grau, segundo a classificação de Kiupel et al. (2011), foram a concentração de células multinucleadas, o número de figuras de mitose e de núcleos bizarros por campo de maior aumento (400x) e a presença de cariomegalia em pelo menos 10\% das células tumorais. Para a graduação em graus I, II ou III, segundo a classificação de Patnaik et al. (1984), levou-se em consideração a extensão tumoral, o pleomorfismo celular e nuclear, a presença de figuras de mitose, células multinucleadas ou células gigantes, mas sem levar em consideração o número específico destas, sendo uma classificação mais subjetiva.

Foi utilizada estatística descritiva para verificar a porcentagem das variáveis analisadas e calculada a média da idade.

\section{Resultados e discussão}

Dos 61 animais avaliados, 36 (59\%) eram machos e 25 (41\%) eram fêmeas. A maior ocorrência de machos já foi descrita em literatura (Kiupel et al., 2005; Costa-Casagrande et al., 2008; Furlani et al., 2008; Navega, 2011), mas há alguns relatos que indicam uma maior incidência de fêmeas (Simoes et al., 1994; Séguin et al., 2001; Berlato et al., 2015). Não se observou diferença significativa entre machos e fêmeas que sugira predisposição ao desenvolvimento do mastocitoma (London \& Seguin 2003; Preziosi et al., 2007; Costa-Casagrande et al., 2008). 
As idades variaram entre 2 e 18 anos, com média de 9,1 anos, similar à descrita em literatura (Costa-Casagrande et al., 2008; Furlani et al., 2008; Romansik et al., 2007; Berlato et al., 2015). Dentre os animais avaliados, três não apresentavam a descrição da idade na ficha. Dos animais com a idade informada, três (5,17\%) tinham entre 0 e 3 anos, 10 (17,24\%) entre 4 e 6 anos, 17 (29,31\%) entre 7 e 9 anos, 22 (37,93\%) entre 10 e 12 anos e 6 (10,35\%) eram maiores que 12 anos.

London \& Seguin (2003) relataram que os animais com nove anos são os mais afetados. No presente estudo, os entre 10 e 12 anos eram os em maior quantidade.

Segundo O'Keefe (1990), animais idosos parecem ser os mais afetados, o que condiz com o nosso estudo, no qual os idosos representaram 73\%. Essa predisposição ao aparecimento tumoral pode estar relacionada à longevidade desses animais, que permanecem expostos a agente cancerígenos por mais tempo (Furlani et al., 2008).

No estudo de Bostock (1986), Thamm \& Vail (2001), Séguin et al. (2001) foram citados animais com mastocitoma de um ano e meio. No presente estudo, não foram encontrados animais jovens (menores que dois anos) com mastocitoma

Foram relatadas 15 raças diferentes. Os cães sem raça definida foram os mais acometidos, representando 24,6\% animais (n=15). Entre os animais de raça definida, os Boxers eram 14 (30,43\%) e os Labradores 7 (14,6\%), assim como o Pinscher (14,6\%). As outras raças encontradas foram Fox Paulistinha, Pit Bull, Golden Retriever, Bichon Frisé, Bernesse, Maltês, Dachshound, Rottweiller, Pastor Alemão, Akita e Pug.

Em relação à raça, os animais sem raça definida e os animais das raças Boxer e Labrador foram aquelas com maior representatividade e esse resultado é similar ao descrito em literatura (Kiupel et al., 2005; Mullins et al., 2006; Costa-Casagrande et al., 2008; Berlato et al., 2015). O grande número de indivíduos sem raça definida encontrados podem ser justificados pelo grande número de atendimento desses animais no Hospital Veterinário da UENF.

Conforme citado em literatura (Thamm \& Vail, 2001; London \& Seguin, 2003), entre os animais de raça definida os da raça Boxer representaram 30,43\% dos animais avaliados. Segundo London et al. (1999), outras raças descendentes do Bulldog (Boston Terrier e Bulldog Inglês) também apresentam predisposição genética, mas não foram observadas no presente estudo.

Ainda segundo Thamm \& Vail (2001), mesmo com a grande presença de mastocitomas em Boxer, geralmente estes são de grau intermediário ou baixo. Este dado foi verificado no presente trabalho, onde, dos 14 Boxers avaliados, 10 (71,5\%) eram de grau I ou II e de baixo grau.

Segundo Murphy et al. (2006), os Labradores tendem a ter tumores mais agressivos. Os achados do presente estudo corroboram com o descrito por estes autores, uma vez que dos sete animais afetados, quatro eram de alto grau.

A raça Pinscher só foi citada uma vez em literatura (Costa-Casagrande et al., 2008) como a mais acometida, mas no presente trabalho ela também apresentou uma alta incidência de mastocitomas. Este fato pode estar associado ao grande número de animais dessa raça atendidos na região de Campos dos Goytacazes.

Em relação à localização tumoral, apenas 52 animais tinham localização tumoral conhecida. Vinte animais (38,5\%) tinham tumor no membro, independente se o membro era torácico ou pélvico, 8 (15,4\%) apresentavam localização genital, 7 (13\%) possuíam tumores no ventre, no dorso ou tumores múltiplos. Também foram encontrados tumores na cabeça, 2 (3,85\%), e no pescoço, $1(1,92 \%)$.

Segundo a literatura, a região mais comum do aparecimento do mastocitoma é o tronco (Séguin et al., 2001; Kiupel et al., 2005; Mullins et al., 2006; Fulcher et al., 2006; Furlani et al., 2008), mas no presente estudos o local mais frequente de aparecimento tumoral foi os membros (38,5\%). A região do tronco (somando-se as regiões do ventre e do dorso) representou 26,9\% dos locais acometidos.

Segundo Govier (2003) e London \& Seguin (2003), a localização inguinal, perineal, prepucial e oral, assim como regiões muco-cutâneas, estão relacionadas a um pior prognóstico. Kiupel et al. (2005) descreveram que os tumores localizados na cabeça e no pescoço aumentam o risco do desenvolvimento de metástase. No presente estudo, dos onze animais com localização genital, na cabeça e no pescoço, todos tiveram tumor de grau intermediário ou indiferenciado (grau II ou III), apenas dois eram de baixo grau. Esses dados corroboram com a literatura (Kiupel et al., 2004; 
Webster et al., 2007), uma vez que, animais com mastocitomas com graus II e III estão relacionados com piores prognósticos.

Por outro lado, observou-se que os mastocitomas localizados em membros apresentaram melhor prognóstico, uma vez que 80\% deles eram de baixo grau. Esses dados corroboram com o descrito por Forrest (1997) e Oliveira (2008) que descrevem que mastocitomas em membros têm comportamento menos agressivo.

A localização disseminada foi observada em $13,4 \%$ dos animais, frequência semelhante a descrita por Govier (2003), Mullins et al. (2006) e Furlani et al. (2008) que descrevem esta apresentação em 9 a 14\% dos animais avaliados. A interferência da presença de múltiplos nódulos no prognóstico dos pacientes não está definida já que há autores que relatam um pior prognóstico associado (Thamm et al., 1999; Séguin et al. 2001; Thamm \& Vail, 2001). No presente estudo, 85,7\% dos tumores disseminados apresentavam baixo grau, o que corrobora com os autores (Thamm et al., 1999; Séguin et al., 2001; Thamm \& Vail, 2001) que não os associam a piores prognósticos.

Em relação à classificação histopatológica, pela classificação descrita por Patnaik et al. (1984), dos 61 tumores avaliados seis (9,8\%) foram considerados classificados como grau I, 45 (73,8\%) grau II e $10(16,4 \%)$ grau III.

A porcentagem de animais com grau I observada neste estudo, foi bem menor do que a descrita em literatura (Simoes et al., 1994; London \& Seguin, 2003; Costa-Casagrande et al., 2008; Furlani et al., 2008; Berlato et al., 2015) que varia entre 20 e 30\%.

Os tumores classificados como grau II foram os mais comuns (73,8\%), corroborando com a literatura (Simoes et al., 1994; London \& Seguin, 2003; Costa-Casagrande et al., 2008; Furlani et al., 2008; Berlato et al., 2015). Essa maior frequência de tumores classificados como grau II ocorre porque há uma tendência dos patologistas a classificarem como grau II os tumores que estão no limite entre o grau I e o grau II. Existe uma variação muito grande entre observadores no momento de classificar um tumor como grau I ou grau II (Kiupel et al., 2011; Garret, 2014). Isso acontece, principalmente, pela presença de critérios subjetivos na classificação proposta por Patnaik et al. (1984) para a diferenciação dos graus.

Pela classificação de Kiupel et al. (2011), dos 61 tumores analisados, 42 (68,85\%) foram classificados como baixo grau e 19 (31,15\%) como alto grau. Esse percentual é similar ao encontrado por Giantin et al. (2012) e Sabattini et al. (2015), contudo os tumores de baixo grau foram encontrados em maior número que o descrito por Takeuchi et al. (2013).

\section{Conclusões}

Com o presente estudo foi possível observar que os mastocitomas grau II são os mais comuns e também os que têm prognóstico mais difícil de ser determinado. Com isso, as variáveis clínicas assumem um papel muito importante na rotina ambulatorial, especialmente a localização, a raça e a idade.

Estudos retrospectivos são importantes para o levantamento de dados nacionais, uma vez que, as características tumorais encontradas no país nem sempre correspondem às descrias na literatura internacional.

\section{Referências}

Berlato, D., Murphy, S., Monti, P., Stewart, J., Newton, J. R., Flindall, A., \& Maglennon, G. A. (2015). Comparison of mitotic index and Ki67 index in the prognostication of canine cutaneous mast cell tumours. Veterinary and comparative oncology, 13(2), 143-150, PMid:23489679.

Bostock, D.E. (1986). Neoplasms of the skin and subcutaneous tissues in dogs and cats. The British Veterinary Journal, 142(1), 1-19. http://dx.doi.org/10.1016/0007-1935(86)90002-3. PMid:3947927.

Costa-Casagrande, T. A., Elias, D. S., Melo, S. R., \& Matera, J. M. (2008). Estudo retrospectivo do mastocitoma canino no serviço de Cirurgia de pequenos animais - Hospital Veterinário da Faculdade de Medicina Veterinária e Zootecnia da Universidade de São Paulo. Archives of Veterinary Science, 13(3), 176-183. http:// dx.doi.org/10.5380/avs.v13i3.11667.

Daleck, C. R., De Nardi, A. B., \& Rodaski, S. Oncologia em cães e gatos (612 p.). São Paulo: Ed. Roca, 2008.

Forrest, L. (1997). Combination cancer treatment: surgery and radiation therapy. Veterinary Medicine, 92(12), 1043-1049. 
Fulcher, R.P., Ludwig, L., Bergman, P., Newman, S. J., Simpson, A. M., \& Patnaik, A. K. (2006). Evaluation of a two-centimeter lateral surgical margin for excision of grade I and Grade II cutaneous mast cell tumors in dogs. Journal of the American Veterinary Medical Association, 228(2), 210-215. http://dx.doi.org/10.2460/ javma.228.2.210. PMid:16426187.

Furlani, J. M., Daleck, C. R., Vicenti, F. A. M., De Nardi, A. B., Pereira, G. T., Santana, A. E., Eurides, D., \& Silva, L. A. F. (2008). Mastocitoma canino: estudo retrospectivo. Ciência Animal Brasileira, 9(1), 242-250.

Garret, L. D. (2014). Canine mast cell tumors: diagnosis, treatment, and prognosis. Veterinary Medicine: Research and Reports., 5, 49-58.

Giantin, M., Vascellari, M., Morello, E. M., Capello, K., Vercelli, A., Granato, A., Lopparelli, R. M., Nassuato, C., Carminato, A., Martano, M., Mutinelli, F., \& Dacasto, M. (2012). c-KIT messenger RNA and protein expression and mutations in canine cutaneous mast cell tumors: correlations with post-surgical prognosis. Journal of Veterinary Diagnostic Investigation, 24(1), 116-126. http://dx.doi.org/10.1177/1040638711425945. PMid:22362941.

Goldschmidt, M. H., \& Hendrick, M. J. (2002). Tumors of the skin and soft tissues. In: D. J. Meuten (Eds.), Tumors in domestic animals (4th ed., pp. 105-109). Iwoa: Iwoa State Press.

Govier, S. M. (2003). Principles of treatment for mast cell tumors. Clinical Techniques in Small Animal Practice, 18(2), 103-106. http://dx.doi.org/10.1053/svms.2003.36624. PMid:12831070.

Kiupel, M., Webster, J. D., Miller, R., \& Kaneene, J. (2005). Impact of tumour depth, tumor location and multiple synchronous masses on the prognosis of canine cutaneous mast cell tumours. Journal of Veterinary Medicine, 52(6), 280-286. http://dx.doi.org/10.1111/j.1439-0442.2005.00726.x. PMid:16050909.

Kiupel, M., Webster, J. D., Bailey, K. L., Best, S., DeLay, J., Detrisac, C. J., Fitzgerald, S. D., Gamble, D., Ginn, P. E. Goldschmidt, M. H., Hendrick, M. J., Howerth, E. W., Janovitz, E. B., Langohr, I., Lenz, S. D., Lipscomb, T. P., Miller, M. A., Misdorp, W., Moroff, S., Mullaney, T. P., Neyens, I., O’Toole, D., Ramos-Vara, J., Scase, T. J., Schulman, F. Y., Sledge, D., Smedley, R. C., Smith, K., W Snyder, P., Southorn, E., Stedman, N. L., Steficek, B. A., Stromberg, P. C., Valli, V. E., Weisbrode, S. E., Yager, J., Heller, J., \& Miller, R. (2011). Proposal of a 2-Tier histologic grading system for canine cutaneous mast cell tumors to more accurately predict biological behavior. Veterinary Pathology, 48(1), 147-155. http://dx.doi.org/10.1177/0300985810386469. PMid:21062911.

London, C. A., Galli, S. J., Yuuki, T., Hu, Z. Q., Helfand, S. C., \& Geissler, E. N. (1999). Spontaneous canine mast cell tumors express tandem duplications in the proto-oncogene c-kit. Experimental Hematology, 27(4), 689-697. http://dx.doi.org/10.1016/S0301-472X(98)00075-7. PMid:10210327.

London, C. A., \& Seguin, B. (2003). Mast cell tumors in the dog. The Veterinary Clinics of North America. Small Animal Practice, 33(3), 473-489, v. http://dx.doi.org/10.1016/S0195-5616(03)00003-2. PMid:12852232.

Mullins, M. N., Dernell, W., Withrow, S., Ehrhart, E., Thamm, D., \& Lana, S. (2006). Evaluation of prognostic factors associated with outcome in dogs with multiple cutaneous mast cell tumors treated with surgery with and without adjuvant treatment: 54 cases (1998-2004). Journal of the American Veterinary Medical Association, 228(1), 91-95. http://dx.doi.org/10.2460/javma.228.1.91. PMid:16426175.

Murphy, S., Sparkes, A., Blunden, A., Brearley, M., \& Smith, K. (2006). Effects of stage and number of tumours on prognosis of dogs with cutaneous mast cell tumours. The Veterinary Record, 158(9), 287-291. http://dx.doi. org/10.1136/vr.158.9.287. PMid:16517820.

Navega, P. R. S. (2011). Mastocitomas em canídeos: estudo retrospecitvo (Dissertação de mestrado). Universidade Técnica de Lisboa, Lisboa.

O'Keefe, D.A. (1990). Canine Mast Cell Tumors. The Veterinary Clinics of North America. Small Animal Practice, 2O(4), 1105-1115. http://dx.doi.org/10.1016/S0195-5616(90)50087-X. PMid:2115219.

Oliveira, J. M. P. (2008). Aspectos patológicos do mastocitoma cutâneo canino - relação com características epidemiológicas e clínicas e seu valor prognóstico (Tese de doutorado). Universidade de Trás-os-Montes e Alto Douro, Vila Real.

Patnaik, A. K., Ehler, W. J., \& MacEwen, E. G. (1984). Canine cutaneous mast cell tumor: morphologic grading and survival time in 83 dogs. Veterinary Pathology, 21(5), 469-474. http://dx.doi.org/10.1177/030098588402 100503. PMid:6435301.

Preziosi, R., Sarli, G., \& Paltrinieri, M. (2007). Multivariate survival analysis of histological parameters and clinical presentation in canine cutaneous mast cell tumours. Veterinary Research Communications, 31(3), 287-296. http://dx.doi.org/10.1007/s11259-006-3427-9. PMid:17195927.

Romansik, E.M., Reilly, C., Kass, P., Moore, P., \& London, C. (2007). Mitotic index is predictive for survival for canine cutaneous mast cell tumors. Veterinary Pathology, 44(3), 335-341. http://dx.doi.org/10.1354/vp.44-3. 335. PMid:17491075

Sabattini, S., Scarpa, F., Berlato, D., \& Bettini, G. (2015). Histologic grading of canine mast cell tumor. Is 2 better than 3? Veterinary Pathology, 52(1), 70-73. http://dx.doi.org/10.1177/0300985814521638. PMid:24513799.

Séguin, B., Leibman, N. F., Bregazzi, V. S., Ogilvie, G. K., Powers, B. E., Dernell, W. S., Fettman, M. J., \& Withrow, S. J. (2001). Clinical outocome of dogs with grade-II mast cell tumors treated with surgery alone: 55 cases (19961999). Journal of the American Veterinary Medical Association, 218(7), 1120-1123. http://dx.doi.org/10.2460/ javma.2001.218.1120. PMid:11318363.

Simoes, J. P. C., Schoning, P., \& Butine, M. (1994). Prognosis of canine mast cell tumors: a comparison of three methods. Veterinary Pathology, 31(6), 637-647. http://dx.doi.org/10.1177/030098589403100602. PMid:7863578. 
Strefezzi, R. F., Xavier, J. G., \& Catão-Dias, J. L. (2003). Morphometry of canine cutaneous mast cell tumors. Veterinary Pathology, 4O(3), 268-275. http://dx.doi.org/10.1354/vp.40-3-268. PMid:12724567.

Takeuchi, Y., Fujino, Y., Watanabe, M., Takahashi, M., Nakagawa, T., Takeuchi, A., Bonkobara, M., Kobayashi, T., Ohno, K., Uchida, K., Asano, K., Nishimura, R., Nakayama, H., Sugano, S., Ohashi, Y., \& Tsujimoto, H. (2013). Validation of the prognostic value of histopathological grading or c-kit mutation in canine cutaneous mast cell tumors: a retrospective cohort study. Veterinary Journal (London, England: 1997), 196(3), 492-498. http:// dx.doi.org/10.1016/j.tvjl.2012.11.018. PMid:23384436.

Thamm, D. H., Mauldin, E., \& Vail, D. (1999). Prednisone and vinblastine chemotherapy for canine mast cell tumor - 41 cases (1992-1997). Journal of Veterinary Internal Medicine, 13(5), 491-497. PMid:10499735.

Thamm, D., \& Vail, D. M. (2001). Mast Cell Tumors. In M. E. Withrow (Eds.), Small Animal Oncology. (261-82). Philadelphia: WB Saunders Company.

Withrow, S. J., \& Vail, D. M. MacEwen's small animal clinical oncology (4th ed.) St. Louis: Saunders Elsevier. 2007.846p

Kiupel, M., Webster, J. D., Kaneene, J. B., Miller, R., \& Yuzbasiyan-Gurkan, V. (2004). The use of KIT and tryptase expression patterns as prognostic tools for canine cutaneous mast cell tumors. Veterinary Pathology, 41(4), 371-337. http://dx.doi.org/10.1354/vp.41-4-371. PMid:15232137.

Webster, J. D., Yuzbasiyan-Gurkan, V., Miller, R. A., Kaneene, J. B., \& Kiupel, M. (2007). Cellular proliferation in canine cutaneos mast cell tumors: associations with c-KIT and its role in prognostication. Veterinary Pathology, 44(3), 298-308. http://dx.doi.org/10.1354/vp.44-3-298. PMid:17491070. 